\title{
A study of the electroluminescence mechanism in a light-emitting composite produced with PEDOT:PSS, PVA and $\mathrm{Zn}_{2} \mathrm{SiO}_{4}: \mathrm{Mn}$
}

\author{
G. Gozzi \\ Physics Department at São Paulo State University - UNESP, 13506-900, Rio Claro, Brazil
}

A R T I C L E I N F O

\section{Keywords:}

Light-emitting device

Electroluminescence mechanism

Light-emitting composite

\begin{abstract}
A B S T R A C T
Light-emitting composites are materials that combine the light-emitting characteristic of phosphor materials with the solubility of conductive polymers. This paper has the purpose of showing an experimental study on electroluminescence in a light-emitting composite comprising PEDOT:PSS as the conductive polymer and $\mathrm{Zn}_{2} \mathrm{SiO}_{4}: \mathrm{Mn}$ as the phosphor material. The relationship between d.c. current and voltage, as well as luminance and voltage, of light-emitting devices were measured for different active layer thickness and different temperature conditions. Luminance was also measured during impedance spectroscopy experiments (a.c. characterization). It was observed that the electroluminescence in the studied light-emitting composite is a field effect mechanism, with turn-on electric field of $(4.6 \pm 0.2) \mathrm{kV} / \mathrm{cm}$, and that the electroluminescence efficiency is independent of temperature. Additionally, it was observed that electroluminescence is strictly dependent on the drift current of charge carriers. Finally, a model was proposed to describe the electrical properties and luminance of the studied light-emitting composite.
\end{abstract}

\section{Introduction}

Phosphor materials are light-emitting inorganic compounds doped with $\mathrm{Mn}, \mathrm{Eu}, \mathrm{Ce}$ and other elements. Since the report of the first lightemitting device, produced with this class of materials by Destriau [1,2], similar studies have been carried out. The main motivation is the wide applicability of some of these phosphor materials, e.g., in a.c. thin film light-emitting devices (ACTFEL) [3], in field emission displays (FED) [4], in cathodic ray tubes [5] (CRT) and in d.c. thin film devices (DCTFEL) [6]. In these materials, electroluminescence occurs as a result of the collision of energetic electrons with the light-emitting centres (light-emitting atoms). This process, called impact excitation, depends mainly on the collision cross section [7] and on the probability of an electron achieving the impact excitation threshold energy [8]. For a relatively high electric field, with magnitude order of $\mathrm{MV} / \mathrm{cm}$, the electrons in a phosphor material achieve sufficient energy to excite the light-emitting atoms [9], which are able to emit light by radiative decay of the excited states.

ACTFEL are light-emitting devices comprised of the phosphor material, placed between two insulating layers, located between electrodes. In this device, the operational mechanism consists of four steps (i) injection of charge carriers through the insulating layers by FowlerNordhein tunnelling; (ii) acceleration of charge carriers by intense electric fields; (iii) impact excitation of light-emitting centres; (iv) radiative decay of excited electron within the light-emitting centres [3]. DCTFEL devices are produced with similar architecture but without the dielectric layers, thus the charge carriers are conducted directly into the light-emitting layer. In these type of device, the operational mechanism consists of three steps: (i) charge transport in the light-emitting material described by $i \propto \exp \left(V^{-1 / 2}\right)$, where $i$ represents the electric current and $V$ the excitation voltage [10-12]; (ii) impact excitation of light-emitting centres; (iii) radiative decay of excited electron in the light-emitting centre. In general, as a consequence of impact excitation [13-15], ACTFEL and DCTFEL devices exhibit turn-on electric fields of $10^{5}-10^{6} \mathrm{~V} / \mathrm{cm}[16-21]$. In several cases, the luminance of the device is proportional to the electric current (for moderate electric fields) [22] and is saturated in high electric field conditions [23,24]. Thus, the efficiency of the device is weakly dependent on the applied electric field or decreases with the increase of the electric field (for high electric fields) $[18,25]$.

After recent advances in conductive polymers research field and the development of phosphor materials, a new light-emitting material was proposed. The material would combine the light-emitting characteristic of the phosphor material $\mathrm{Zn}_{2} \mathrm{SiO}_{4}$ :Mn with the solubility of conductive polymers to fabricate a new type of solution-processed light-emitting device [26]. In this study, a colloidal solution was prepared by dissolving a polymeric conductive material in organic solvent and adding micro-particles of the phosphor material. The colloidal solution was

E-mail address: giovanigozzi@rc.unesp.br. 

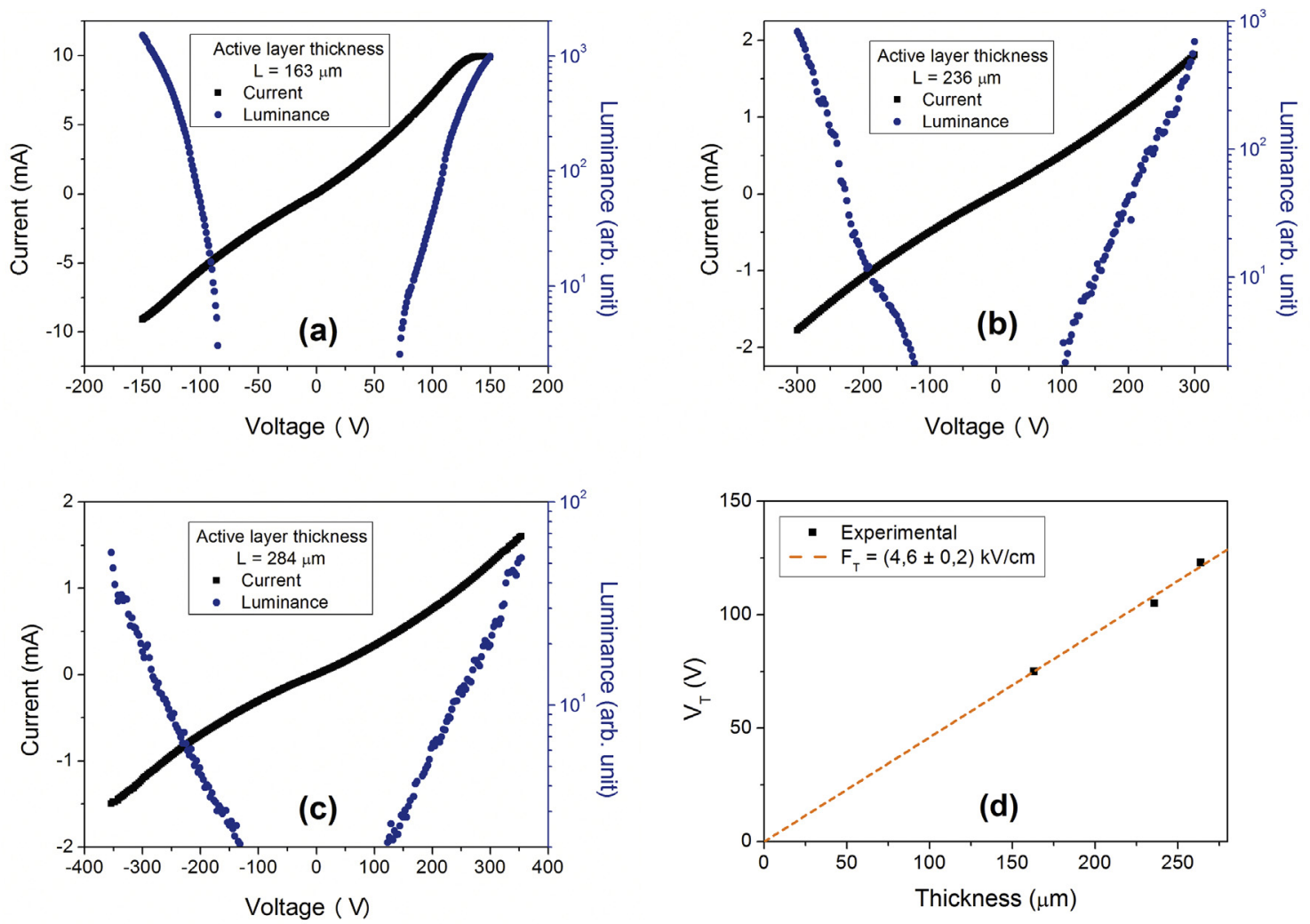

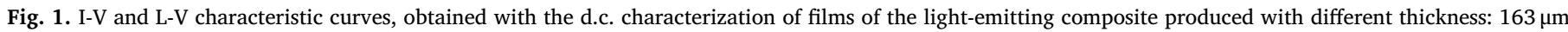
(a), $236 \mu \mathrm{m}$ (b), $284 \mu \mathrm{m}$ (c), (d) Light-emitting composite turn-on voltage as a function of the film thickness.

used to coat glass substrates containing a transparent and conductive electrode in order to produce a film with the light-emitting composite. A metallic electrode was deposited over the active layer to finalize the fabrication of the electroluminescent device produced with a lightemitting composite (LECEL - Light-Emitting Composite Electroluminescent Device). The fabrication of LECEL is advantageous because solution-processed materials can be processed by simple printing or coating methodologies, such as screen-printing [27], spray-coating [28], spin-coating [29], drop-casting [8], and roll-to-roll [30].

Some studies on the electrical properties of LECEL show that the operation of this device depends on two main processes: (i) charge injection from electrodes to the active layer and (ii) charge transport in the light-emitting composite. Charge injection has been described as a thermal-stimulated process. Thus, for light-emitting composites produced with p-doped semiconductor polymer, it was observed that nonreactive electrodes, such as gold or indium thin oxide (ITO), are the best materials to fabricate LECEL [31]. Regarding charge transport in the light-emitting composite, two different mechanisms may play important roles: the charge transport in the conductive phase, and the concentration of micro-particles in the phosphor material [32].

However, electroluminescence mechanism in these type of devices is not completely understood. In this sense, the present paper investigates electroluminescence of LECEL produced with poly(3,4-ethylenedioxythiophene):polystyrene sulfonate (PEDOT:PSS) as conductive polymer and $\mathrm{Zn}_{2} \mathrm{SiO}_{4}: \mathrm{Mn}$ as electroluminescent phosphor material. The devices were characterized using both d.c. and a.c. excitation methodology, and the results have shown that LECEL electroluminescence is dependent on excitation voltage, active layer thickness, a.c. signal frequency and temperature. These results were used to build a model to describe the electroluminescence of LECELs as a field-effect mechanism.

\section{Material and methods}

The experimental details are shown in two sections, one describing the fabrication of the devices (section 2.1) and another describing the characterization apparatus (section 2.2).

\subsection{Device fabrication}

The devices were produced following three main steps: (i) preparation of the light-emitting composite solution; (ii) deposition of the active layer film; (iii) deposition of gold top electrodes. The lightemitting composite solution was prepared with three different materials: polyvinyl alcohol (PVA), $\Sigma$ - Aldrich ( $\mathrm{M}_{\mathrm{w}} 89000-98000$, 99\% hydrolyzed), poly(3,4-ethylenedioxythiophene):polystyrene sulfonate (PEDOT:PSS), commercialized as Clevios ${ }^{\mathrm{TM}} \mathrm{PH} 1000$ and purchased from Heraeus, and $\mathrm{Zn}_{2} \mathrm{SiO}_{4}: \mathrm{Mn}$ powder, with micrometric particles, purchased from Fluka. Some fundamental characteristics of these materials, such as crystalline structure, morphological, chemical, electrical and optical properties, can be found in references [33-40].

The PEDOT:PSS was sourced in water solution at a concentration of $30 \mathrm{mg} / \mathrm{ml}$. In our experiment, 5\% (volume) of ethylene glycol was added to the PEDOT:PSS solution in order to achieve high-conductivity grade [33]. The PEDOT:PSS solution was blended with a solution of PVA in water, also at $30 \mathrm{mg} / \mathrm{ml}$. PVA is a high transparency polymer used to control polymeric blend conductivity and transparency [34] and to improve mechanical stability [35]. The blend composition was established at a weight ratio of (90/10\%-wt) of PEDOT:PSS/PVA. This composition exhibits electric conductivity of $210^{-5} \mathrm{~S} / \mathrm{cm}$ and low light absorption coefficient, $0.6 \mu \mathrm{m}^{-1}$ [34]. The light-emitting composite was obtained by adding $\mathrm{Zn}_{2} \mathrm{SiO}_{4}: \mathrm{Mn}$ micro-particles to the polymeric blend solution at a weight ratio of $90 \%$-wt of inorganic material and 10 $\%$-wt of polymeric blend. This solution was stirred for $24 \mathrm{~h}$ at room conditions until a homogeneous solution of the light-emitting 
composite was obtained.

Glass substrates containing patterned ITO electrodes were coated with the light-emitting composite solution by drop casting. After that, the samples were heated at $80^{\circ} \mathrm{C}$, in an oven, for $24 \mathrm{~h}$, to promote solvent evaporation. Finally, gold electrodes were deposited on the top surface of the active layer. Top electrodes were produced with thermally evaporated gold using an Edwards system (model AUTO-306). Therefore, LECELs were produced employing Ohmic contacts, produced with gold and ITO, with negligible electrical resistance [31]. The active area of the device was $1 \mathrm{~cm}^{2}$.

\subsection{Experimental set-ups}

Measurements were performed using a Janis cryostat (model VPF 100 ) with operational pressure of $10^{-2} \mathrm{mbar}$ and temperature controlled by a LakeShore controller (model 325).

D.C. Characterization: d.c. characterization was performed using a source/meter unit (Keithley 2410) as voltage source and ammeter. A silicon photo-diode coupled to an electrometer (Keithley 6517) was used to measure the electroluminescence of the devices in arbitrary unit.

A.C. Characterization: a.c. characterization was performed using a Gegem function generator (model PU-222), and a Trek signal amplifier (model 10/10B) as the voltage source. Electric currents were measured using a reference resistor (with electrical resistance significantly lower than the impedance of the devices) and a Minipa oscilloscope (model 1221S). The rms luminance value was measured using a silicon photodiode coupled to an electrometer (Keithley 6517). A.c. characterization was performed employing voltage signals with diverse frequencies and fixed amplitude of $350 \mathrm{~V}$.

Film thickness was measured with a Digimess digital micrometer, with resolution of $1 \mu \mathrm{m}$.

\section{Results}

In the present study, we performed the electro-optical characterization of a light-emitting composite using d.c. and a.c. excitation of LECELs with Ohmic contacts, for different active-layer thickness in diverse temperature conditions. Section 3.1 shows the results obtained from d.c. characterization of LECELs produced with different active layer thickness. Section 3.2 shows the results obtained from d.c. characterization of a LECEL in diverse temperature conditions. Finally, section 3.3 shows results obtained from a.c. characterization of a LECEL in diverse temperature conditions.

\subsection{D.C. Characterization of LECELs with different active layer thickness}

Fig. 1 shows the d.c. current vs. voltage (I-V) and the luminance vs. voltage (L-V) characteristic curves of light-emitting composite films produced with different thickness. I-V curves exhibit an almost linear behavior, while L-V curves exhibit an exponential behavior. The I-V results indicate the light-emitting composite behaves as a conductive material, with properties quite similar to the polymeric blend used as a conductive matrix in the production of the light-emitting composite [34]. The exponential behaviour of L-V curves of the light-emitting composite was equivalent to that of ACTFEL devices [41].

The voltages employed to turn on the electroluminescence in the studied light-emitting composite, $V_{\mathrm{T}}$, were dependent on the film thickness. Fig. 1 (d) shows that $V_{\mathrm{T}}$ and film thickness are linearly dependent, indicating a field effect process. The turn-on electric field, obtained from data shown in Fig. 1(d), was $(4.6 \pm 0.2) \mathrm{kV} / \mathrm{cm}$.

Fig. 2(a) shows that electroluminescence efficiency correlates with the inverse of the electric field. For electric fields close to the turn-on value, an exponential-like behavior was identified, $\eta(F) \propto \exp \left(-\frac{F_{0}}{F}\right)$, where $F$ represents the excitation electric field and $F_{0}$ an empiric field factor. It is possible to verify, in Fig. 2(a), an intersection of the fittings in the value corresponding to the turn-on electric field. Fig. 2(b) shows that the field factor increases as active layer thickness decreases. This result indicates that thinner films of light-emitting composites exhibit stronger increase in efficiency with the increase of the electric field than thicker films. Additionally, experimental data obtained from the thinnest light-emitting composite film exhibited an almost exponential behavior over all experimental range. For electric fields significantly higher than the turn-on value, thicker light-emitting composite films exhibited electroluminescence efficiency that surpasses the exponential behavior.

\subsection{D.C. Characterization in diverse temperature conditions}

I-V and L-V characteristic curves of the light-emitting composite in diverse temperature conditions are depicted in Fig. 3(a) and (c), which show that I-V curves correlate with temperature (Fig. 3(a)). Analysis were performed using the 3-D variable range hopping model (3D-VRH) [42], which describes the charge transport in disordered materials, in order to obtain the hopping temperature of charge transport in the light-emitting composite for different excitation voltages.

Fig. 3(b) shows the hopping temperature, $T_{0}$, for charge transport in the light-emitting composite in comparison with the conductive matrix (as shown in the appendix, figure A1(b)). Hopping temperatures of charge transport in the light-emitting composite varied between $0.510^{5}$
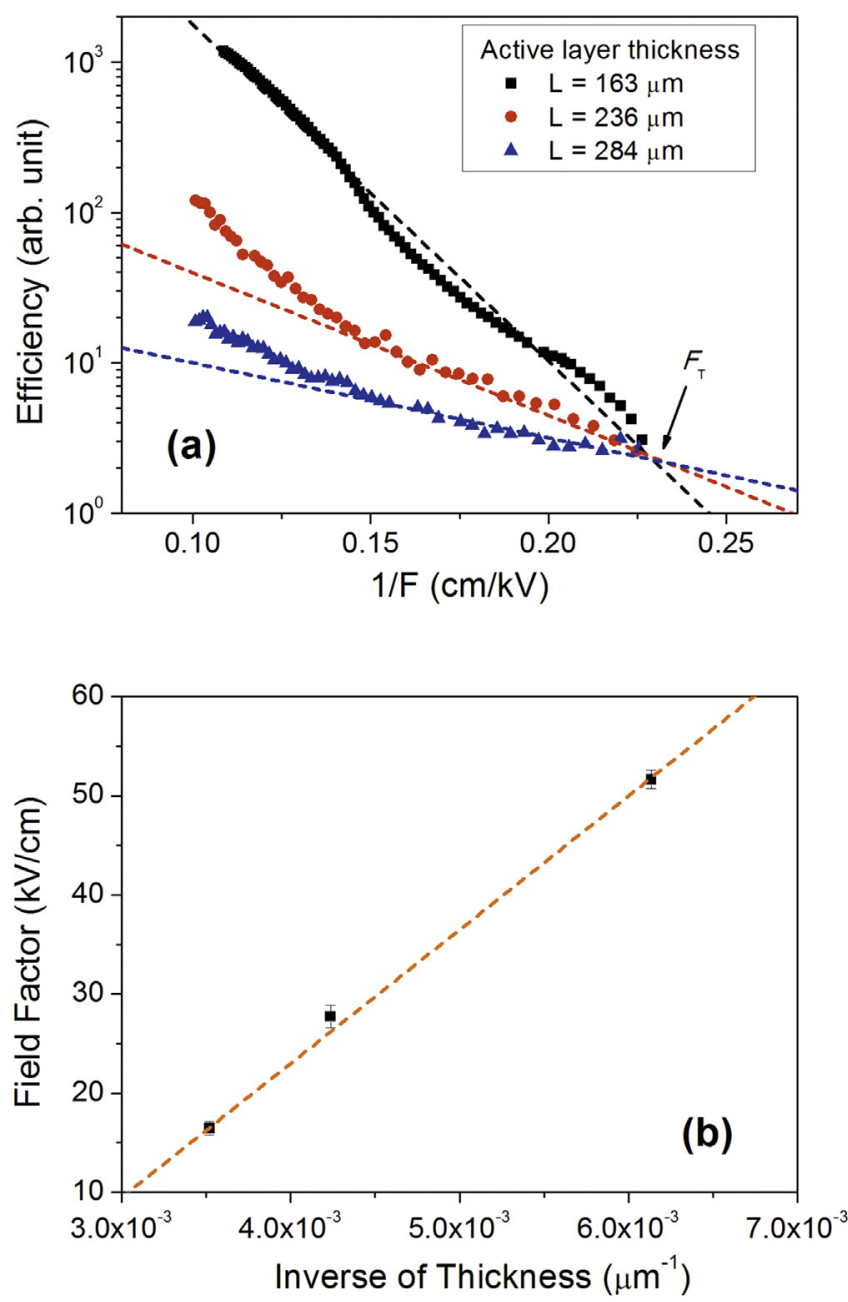

Fig. 2. (a) Light-emitting composite electroluminescence efficiency as a function of the inverse of the electric field. (b) Field factor as a function of the inverse of the film thickness. 

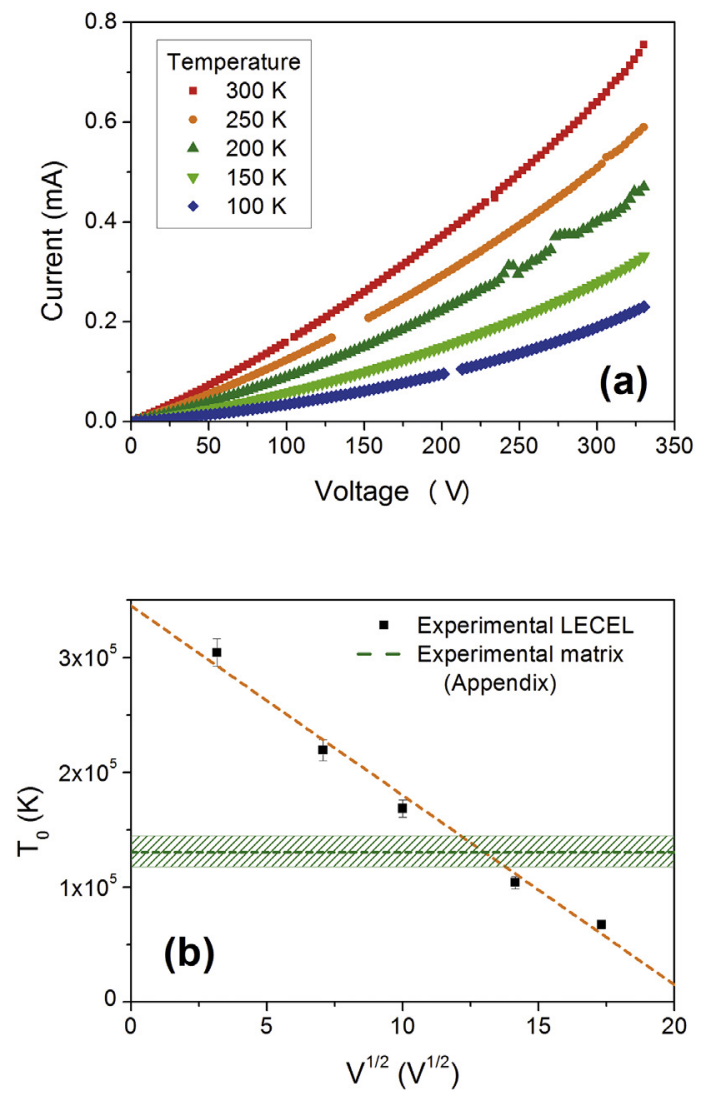
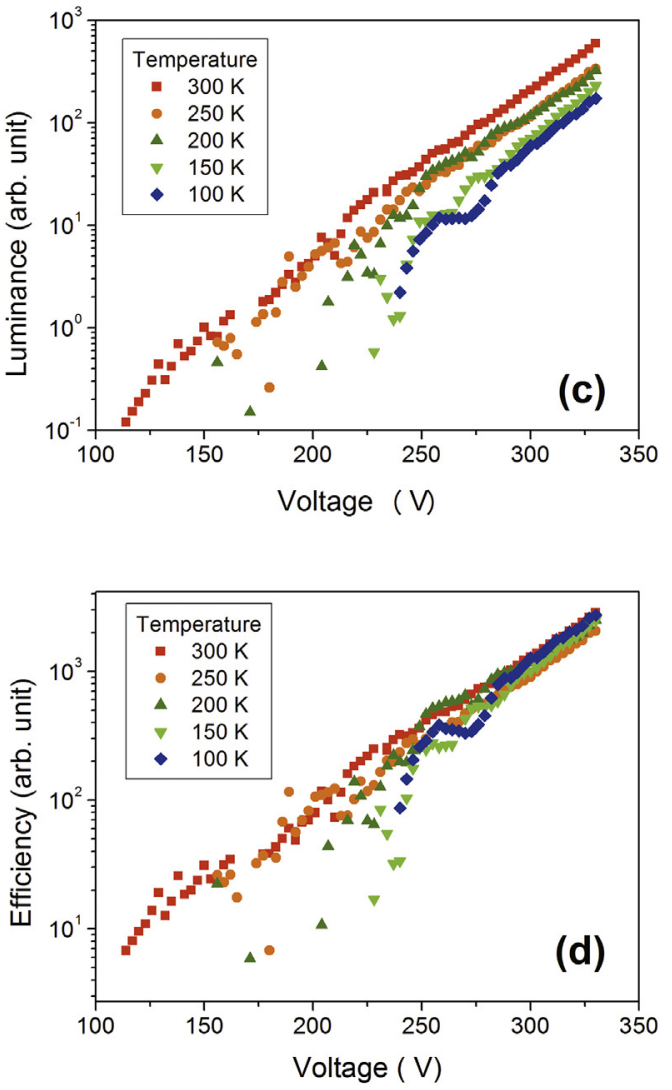

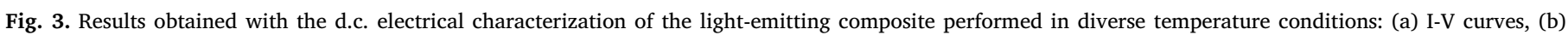
hopping temperatures, (c) L-V curves and (d) electroluminescence efficiency as a function of the excitation voltage.

and $3.010^{5} \mathrm{~K}$. Regarding the conductive matrix (PEDOT:PSS/PVA (10/ $90 \%$-wt) blend), the hopping temperature of charge transport exhibited the same order of magnitude: $(1.3 \pm 0.1) 10^{5} \mathrm{~K}$.

$\mathrm{L}-\mathrm{V}$ characteristic curves, shown in Fig. 3(c), exhibit almost the same dependence on temperature as the I-V curves. This correspondence can be verified in Fig. 3(d), in which electroluminescence efficiency is plotted as a function of excitation voltage. These results show that electroluminescence efficiency in the studied light-emitting composite does not depend on temperature.

\subsection{A.C. Characterization in diverse temperature conditions}

A.c. currents in the light-emitting composite were measured for voltage signals with different frequencies and fixed amplitude (350 V). Data analysis was performed considering two components of the electric current, one in phase with the applied voltage (real component, Re $\left.\left[i^{*}(f)\right]\right)$ and another with a $-\frac{\pi}{2}$ phase (imaginary component, -Im $\left.\left[i^{*}(f)\right]\right)$. The real component of the electric current describes the drift current in the light-emitting composite, while the imaginary component describes displacement currents. Fig. 4 depicts the components of the electric current and the electroluminescence rms value obtained in diverse temperature conditions, as a function of the excitation signal frequency.

The imaginary component of the electric current shows a typical capacitive behavior, $-\operatorname{Im}\left[i^{*}(f)\right]=-\operatorname{Im}\left[C \frac{d}{d t} V^{*}(t)\right] \propto 2 \pi f C$. Where $C$ represents the LECEL capacitance, $V^{*}(t)$ the applied voltage signal and $i *(f)$ the complex electric current. Since the capacitance of the devices is not dependent on temperature, the displacement current is also independent of temperature, as noticed in Fig. 4. For an ideal conductor, the drift current (the real component of the complex electric current) is independent of the excitation signal frequency. However, in disordered materials, for high frequencies, short range charge transport (localized motion) occurs. In this condition, mobility of the charge carrier is increased due to a reduction in the number of potential barriers that opposes charge motion [32,43]. As a consequence, an increase in the drift current occurs with the increase of the excitation signal frequency, as exhibited by the light-emitting composite in Fig. 4. Moreover, we observed that the characteristic frequency of charge transport in the light-emitting composite exhibits the same order of magnitude than that of the conductive matrix (as shown in the appendix, figure A1(a)). This comparison indicates that charge transport in the light-emitting composite occurs mainly in the conductive phase, with weak influence of the inorganic phase.

Fig. 4 also shows the dependence of electroluminescence rms value as a function of the excitation signal frequency for different temperature conditions. Regarding frequencies higher than the characteristic frequency, a.c. electroluminescence efficiency exhibits a drastic decrease as frequency increases in all temperature conditions (Fig. 4). As discussed before, in this condition, the drift electric current originates from short range charge transport. Thus, the results presented in Fig. 4 show that the light-emitting composite exhibits high electroluminescence efficiency under long-range charge transport conditions, and that electroluminescence is drastically diminished under shortrange charge transport conditions. In summary, the comparison between electric current and electroluminescence efficiency of the lightemitting composite, obtained with a.c. excitation in diverse temperature conditions, indicates that the electroluminescence mechanism in the studied composite is strongly dependent on the long-range drift currents of charge carriers.

\section{Discussion}

The studied light-emitting composite was produced with insulating micro-particles dispersed in a conducting matrix as sketched in Fig. 5. 

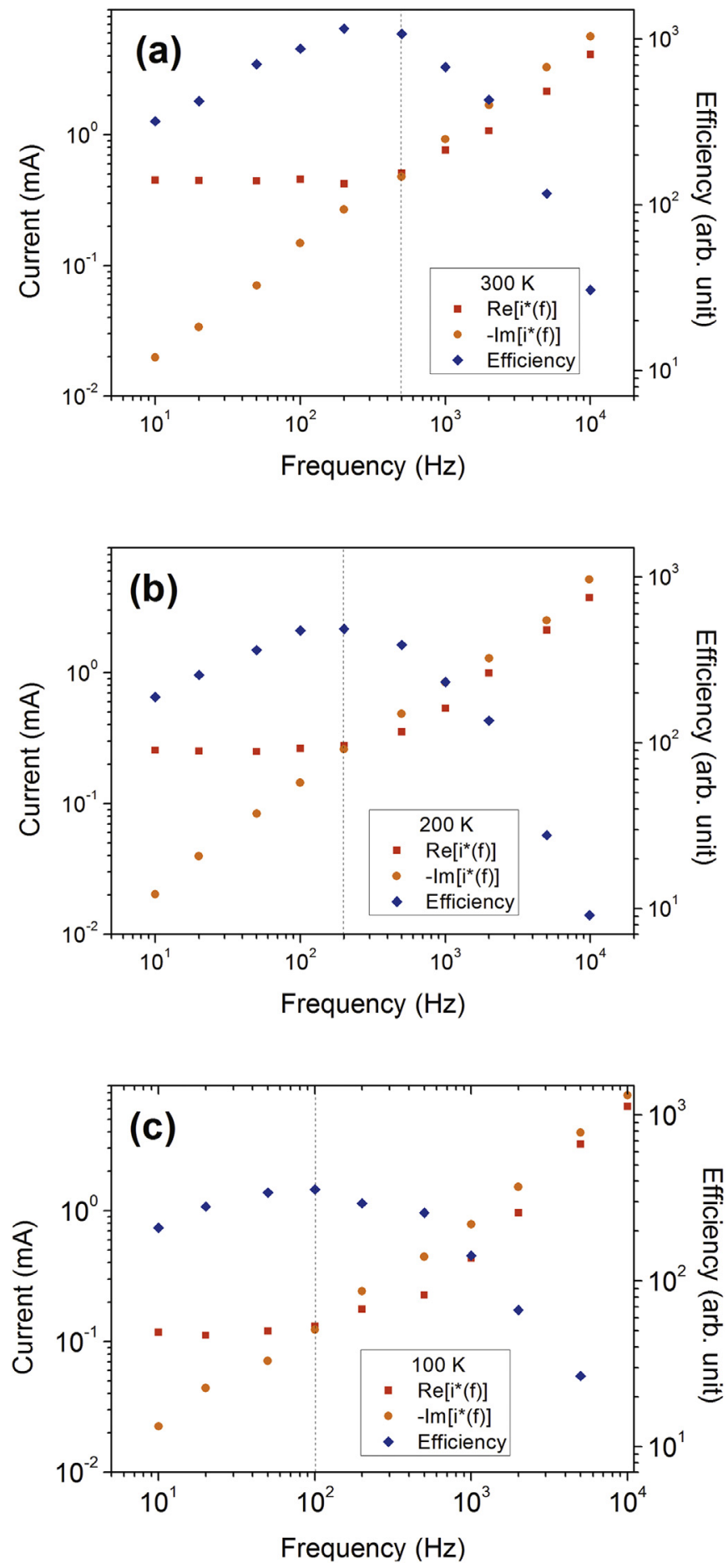

Fig. 4. Real and imaginary components of the electric current and the rms value of the electroluminescence efficiency as a function of the frequency obtained in diverse temperature conditions: (a) $300 \mathrm{~K}$, (b) $200 \mathrm{~K}$ and (c) $100 \mathrm{~K}$.

Even though the weight concentration of the insulating phase was much higher than that of the conductive matrix, it is reasonable to consider the presence of conductive channels (represented as a black line in Fig. 5), where the conductive matrix is percolated [32,44], i.e., insulating micro-particles are absent. On the other hand, in non-percolated channels (represented as a red line in Fig. 5), insulating microparticles are present and charge transport is limited by the insulating phase [32]. As charge transport occurs both in percolated and in non-

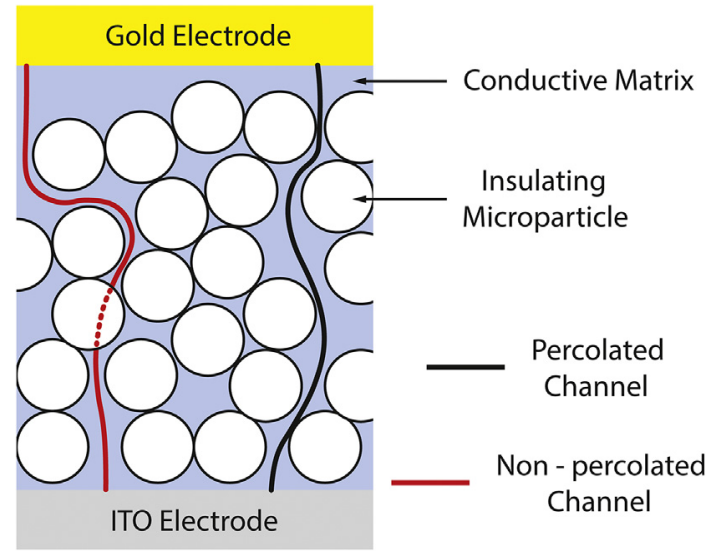

Fig. 5. Sketch of the material morphology, where the black line represents a percolated channel and the red line represents a non-percolated channel. (For interpretation of the references to colour in this figure legend, the reader is referred to the Web version of this article.)

Table 1

Electrical resistivity of the light-emitting composite obtained of films with different thickness.

\begin{tabular}{ll}
\hline Active Layer Thickness $(\mu \mathrm{m})$ & Active Layer Resistivity $(\mathrm{k} \Omega . \mathrm{cm})$ \\
\hline 163 & 1.0 \\
236 & 7.1 \\
284 & 8.4 \\
\hline
\end{tabular}

percolated channels, it is reasonable to describe the total electric current in the light-emitting composite as the summation of the electrical current over all channels.

We observe, in Figs. 1 and 3, an almost linear dependence of the electrical current on the applied voltage. Furthermore, the hopping temperature for charge transport in the light-emitting composite was approximately the same than that of the conductive matrix. These results indicate the electric current in the light-emitting composite is dominated by charge transport in the conductive matrix. Nevertheless, the influence of the inorganic material on the electrical properties of the light-emitting composite is not negligible because these insulating micro-particles inhibit the establishment of percolated channels.

It is reasonable to suppose that the density of percolated channels per unit of the device active area decreases as the number of insulating micro-particles stacked over that unit of area increases. Thus, we could expect an increase in the composite electrical resistivity with the increase of inorganic micro-particles weight concentration [32]. In addition, one would expect a decrease in the density of percolated channels as film thickness increases, because in thicker films a higher amount of insulating particles decant over a unit of active area. This consideration is confirmed by the results in Table 1, which shows that the electrical resistivity of the light-emitting composite, obtained from the results presented in Fig. 1(a), (b) and (c), increases with active layer thickness.

Charge transport in non-percolated channels can be described as the conduction of charge carriers through the conductive matrix up to the edges of an insulating particle or of a small number of insulating particles. For low voltages, charge carriers likely accumulate in the vicinity of the inorganic particles, where the electric field is increased. For higher voltages, the electric field across the inorganic particles (effective electric field) can be sufficient to promote charge injection in the inorganic micro-particles, where electroluminescence occurs. The electroluminescence spectrum of the light-emitting composite, shown 
in figure A2 (Appendix), exhibits a peak at $530 \mathrm{~nm}$ and is quite similar to that of $\mathrm{Zn}_{2} \mathrm{SiO}_{4}: \mathrm{Mn}$ [21], confirming that the electroluminescence of the studied composite occurs on the insulating micro-particles. Thus, electroluminescence is likely promoted by charge carriers conducted only by non-percolated channels, being injected in the insulating microparticles.

Considering the device turn-on voltage as $10^{2} \mathrm{~V}$ (as shown in Fig. 1) and the size of a small number of micro-particles as $10^{-4} \mathrm{~cm}$, it is possible to estimate the effective turn-on electric field as $10^{6} \mathrm{~V} / \mathrm{cm}$, which is consistent with the turn-on electric field in DCTFEL and ACTFEL [16-21]. For effective electric fields of $10^{6} \mathrm{~V} / \mathrm{cm}$ magnitude order or higher, we consider that charge carriers accumulated in the edges of the insulating micro-particles are injected and promote electroluminescence by impact excitation of luminescent centers. As shown in Fig. 3, electroluminescence efficiency of the LECEL is independent of temperature, indicating the charge injection mechanism in the insulating particles is also independent of temperature. Considering this evidence, we assume that charge injection in the insulating material occurs by Fowler-Nordheim tunnelling, as described for ACTFEL [3]. Thus, equation (1) describes current density for charge injection in an insulating single particle, $J_{s p}(F)$.

$J_{s p}(F)=\frac{\alpha}{\phi} F^{2} \operatorname{Exp}\left[-\frac{\beta \phi^{3 / 2}}{F}\right]$

Where $F$ represents the electric field across the particle, $\phi$ represents the magnitude of the potential barrier, $\alpha$ and $\beta$ are constants with typical orders of magnitude of $10^{-6} \mathrm{~A} . \mathrm{eV}^{\mathrm{V}} \mathrm{V}^{-2}$ and $10^{3} \mathrm{eV}^{-3 / 2} . \mathrm{V} . \mu \mathrm{m}^{-1}$ respectively.

Considering that the active layer of a LECEL contains several nonpercolated channels, each one containing a different number of insulating particles, a distribution of effective thickness of insulating layers, $w$, must be taken into account in the calculation of the total current density in the non-percolated phase, as shown in equation (2-a).

$J(V) \propto \int_{w_{\min }}^{\infty} \frac{\alpha}{\phi}\left(\frac{V}{w}\right)^{2} \operatorname{Exp}\left[-\frac{\beta \phi^{3 / 2}}{(V / w)}\right] g(w) d w$

$g(w)=\frac{1}{\sqrt{2 \sigma^{2} \pi}} \operatorname{Exp}\left[-\frac{\left(w-w_{0}\right)^{2}}{2 \sigma^{2}}\right]$

Where $V$ represents the excitation voltage, $w_{\min }$ the minimum value of the effective thickness for the insulating layer and $g(w)$ a Gaussian distribution of effective thickness with mean value, $w_{0}$, and variance, $\sigma^{2}$, as explicited in equation (2-b).

Considering that the luminous efficiency of the phosphor material is weakly dependent on the applied voltage (or electric field), the luminance of the light-emitting composite can be considered as proportional to the charge-injecting current in the insulating layers, $L(V) \propto J(V)$. Fig. 6(a) shows a comparison between experimental data and a simulation of the luminance vs. voltage curves considering the model described by equation (2), where typical values were assigned to $\alpha$ and $\beta$, $\phi$ was considered as $3 \mathrm{eV}$ and $w_{\min }$ as $1 \mu \mathrm{m}$. We also considered a Gaussian distribution of effective thickness of insulating layers as shown in Fig. 6(b).

The simulations shown in Fig. 6(a) are in agreement with experimental data, mainly in forward bias regime, were the ITO electrode was operated as anode and the gold electrode as cathode. Additionally, the mean value of the effective thickness of the insulating layer was proportional to the active layer thickness, which corroborates the result shown in Fig. 1(d), where the turn-on voltage was proportional to active layer thickness. Once the electric current in non-percolated
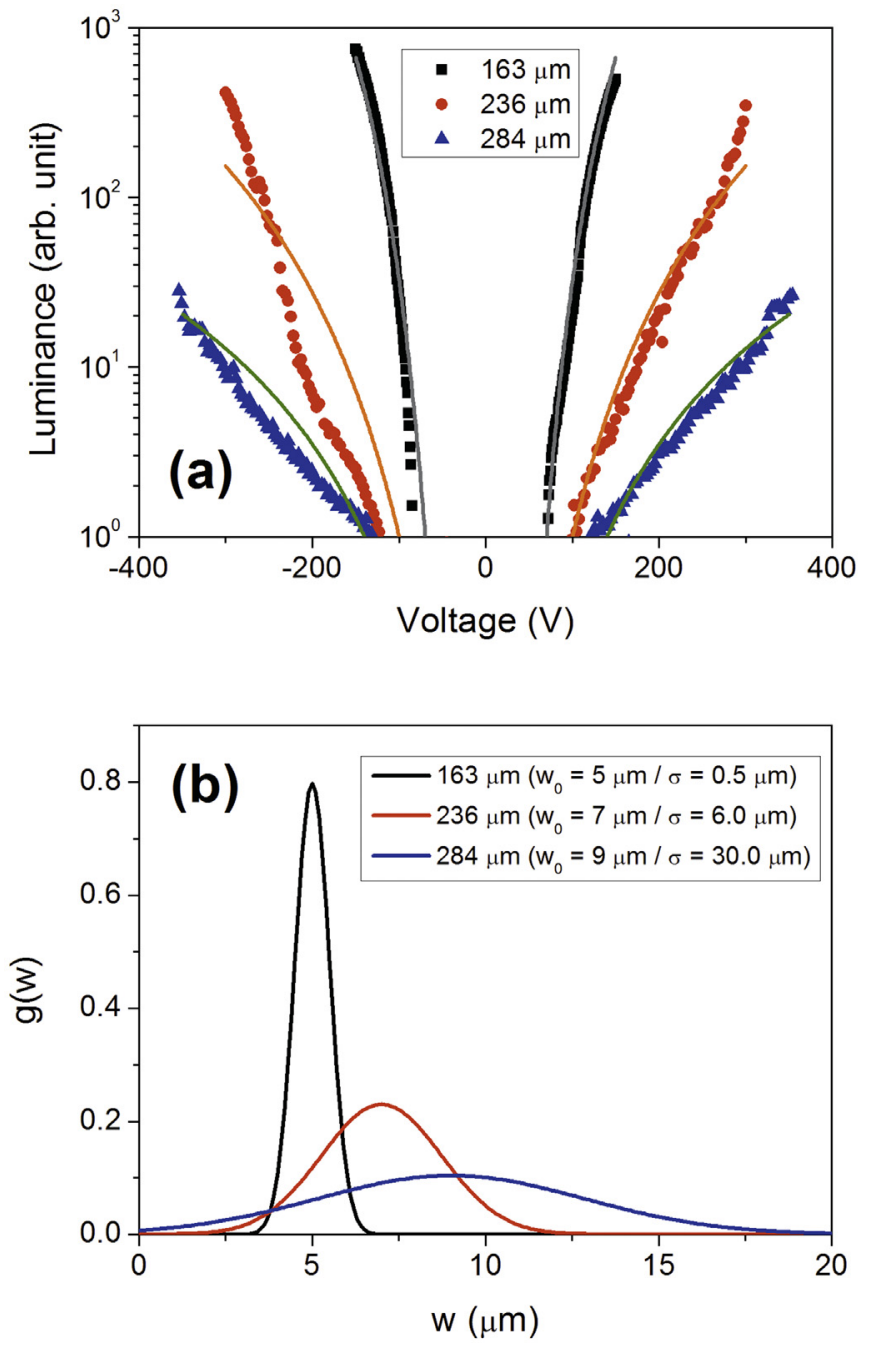

Fig. 6. A comparison of the experimental data with simulations of the lightemitting composite luminance using the model described by equation (2): (a) Experimental and theoretical L-V curves; (b) Gaussian distributions of effective thickness of insulating layers.

channels of the light-emitting composite decreases as the effective thickness of insulating layers increases, as shown in equation (2), our model helps shed light on the reason why the efficiency of the devices increases as active layer thickness decreases, as shown in Fig. 2(a).

Considering that, for lower voltages, light emission is turned on in non-percolated channels with low effective thickness of insulating layers, equation (3) is an approximation to describe the dependence of the composite luminance on electric fields close to the turn-on limit.

$L(F) \propto \frac{\alpha}{\phi} F^{2} \operatorname{Exp}\left[-\frac{\beta \phi^{3 / 2}}{F} \frac{w_{\min }}{d}\right]$

Where $d$ represents the sample thickness. Considering, additionally, that the electric current in the light-emitting composite is mainly due to charge transport in percolated channels, the approximation $I \propto F$ is valid because of the almost Ohmic behavior of the current vs. voltage curves shown in Fig. 1. In this case, the electrical power consumption of the light-emitting composite is $P \propto F^{2}$. Taking into account these approximations, electroluminescence efficiency of the light-emitting 
composite can be described by equation (4).

$\eta\left(F_{T}\right) \propto \operatorname{Exp}\left[-\frac{\beta \phi^{3 / 2}}{F} \frac{w_{\min }}{d}\right]=\operatorname{Exp}\left[-\frac{F_{0}}{F}\right]$

Where the field factor is $F_{0}=\beta \phi^{3 / 2} \frac{w_{\text {min }}}{d}$. The experimental data presented in Fig. 2(b) shows this linear dependence of the field factor on the inverse of sample thickness. In addition, it was possible to estimate $w_{\text {min }}$ from the linear fit shown in Fig. $2(\mathrm{~b})$ as $(0.3 \pm 0.1) \mu \mathrm{m}$. However, for thicker light-emitting composite films and for electric fields significantly higher than the turn-on limit, there is a discrepancy between equation (4) and the experimental data i.e. electroluminescence efficiency surpasses the exponential behavior described in equation (4), as shown in Fig. 2(a).

For the thinnest light-emitting composite film, data simulation indicates a narrow distribution of effective thickness of the insulating layer. In this case, it is possible to approximate the Gaussian distribution of effective thickness by a mean value, $\bar{w}$. Considering this approximation, the efficiency of the light-emitting composite may be described by $\eta(F) \propto \operatorname{Exp}\left[-\frac{\beta \phi^{3 / 2}}{F} \frac{\bar{w}}{d}\right]=\operatorname{Exp}\left[-\frac{F_{0}}{F}\right]$. This means electroluminescence is achieved approximately at the same applied voltage in all non-percolated channels. For thicker films, which exhibit a broad distribution of effective thickness of the insulating layer, electroluminescence in different non-percolated channels is achieved with different applied voltages. In this case, equation (4) is adequate to describe the efficiency of the light-emitting composite only for electric fields close to the turn-on value. For electric fields higher than the turnon value, electroluminescence occurs at a higher number of non-percolated channels, indicating the origin of the discrepancy between equation (4) and the experimental data for films with a broad distribution of effective thickness of insulating layers.

\section{Conclusions}

We produced LECEL devices with different active layer thickness and Ohmic contacts to study the electroluminescence mechanism in light-emitting composites produced with PEDOT: PSS, PVA and
$\mathrm{Zn}_{2} \mathrm{SiO}_{4}: \mathrm{Mn}$. These devices were characterized with respect to their electrical properties and light output in steady-state (d.c.) regime and when excited with a.c. signal. It was shown that the electroluminescence of the produced light-emitting composites is a field effect mechanism, with turn-on electric field $(4.6 \pm 0.2) \mathrm{kV} / \mathrm{cm}$. We have also shown that electroluminescence efficiency exhibits an exponential dependence on the electric field and is approximately independent of temperature. Experimental data was analyzed considering the lightemitting composite as a biphasic material, containing conductive channels, composed only by the conductive matrix, and non-percolated channels, where the inorganic light-emitting particles limit charge flow. Charge transport in the active material was described as the summation of the electrical current over all percolated (conductive) and non-percolated (insulating) channels. Considering this model, the influence of non-percolated channels on the electrical properties of the light-emitting composite is weak in comparison to that of the conductive channels, in accordance to the experimental data.

Electroluminescence likely occurs in the inorganic insulating lightemitting particles, i. e., in the non-percolated channels. In non-percolated channels, charge carriers are conducted up to the edges of the inorganic particles, which act as insulating layers. With the increase of the external voltage, the effective electric field in the vicinity of the inorganic particles achieves $10^{5}-10^{6} \mathrm{~V} / \mathrm{cm}$, leading to charge injection in the insulating material, triggering electroluminescence. Charge injection in the insulating layers was described by Fowller-Nordheim tunnelling and a mathematical model was proposed to simulate the experimental data. The mathematical model and the experimental data showed good agreement, and helped shed light on the influence of constructive parameters, such as active layer thickness, on the luminous efficiency of a LECEL.

\section{Acknowledgments}

The author would like to thank professors Dante Luis Chinaglia and René Armando Moreno Alfaro for the discussions; and Larissa Galante Elias for the proof reading. This work was supported by the São Paulo Research Foundation, FAPESP [grant number 2012/19005-0].

\section{Appendix}

This section shows complementary results, such as the electrical characterization of the material used as conductive matrix of the light-emitting composite. Figure A.1(a) depicts the complex conductivity of this material as a function of a.c. excitation voltage frequency. In this figure, for frequencies lower than $300 \mathrm{~Hz}$, the real component of conductivity is independent of the frequency, with $210^{-5} \mathrm{~S} / \mathrm{cm}^{\circ}$ of magnitude, and is more intense than the imaginary component of the conductivity. This result indicates that for frequencies lower than $300 \mathrm{~Hz}$, charge transport in the conductive material is carried by drift currents (in phase with the excitation voltage). For frequencies higher than $300 \mathrm{~Hz}$, the imaginary component of the electrical conductivity surpasses the real component. This result indicates that, in high frequency range, charge carriers are not conducted in phase with the excitation signal, i. e., the long-range drift current is decreased and the displacement current is increased.

Figure A.1(b) shows the electrical conductivity of the conductive material in the d.c. limit (extracted from the real component in frequencyindependent limit) as function of temperature in a 3D Variable Range Hopping (VRH) plot. In this figure, experimental data was fitted by the 3D-VRH equation, equation (A.1), and the hopping temperature was obtained as $(1.3 \pm 0.1) 10^{5} \mathrm{~K}$.

$\sigma_{d c}(T) \propto \exp \left[-\left(\frac{T_{0}}{T}\right)^{1 / 4}\right]$

Where $T$ represents the experimental temperature and $T_{0}$ the hopping temperature.

Figure A.2 shows the electroluminescence spectra of the light-emitting composite with an emission peak in $530 \mathrm{~nm}$. This wavelength is close to that of $\mathrm{Zn}_{2} \mathrm{SiO}_{4}: \mathrm{Mn}(524 \mathrm{~nm})$, indicating that electroluminescence occurs in the inorganic micro-particles. 

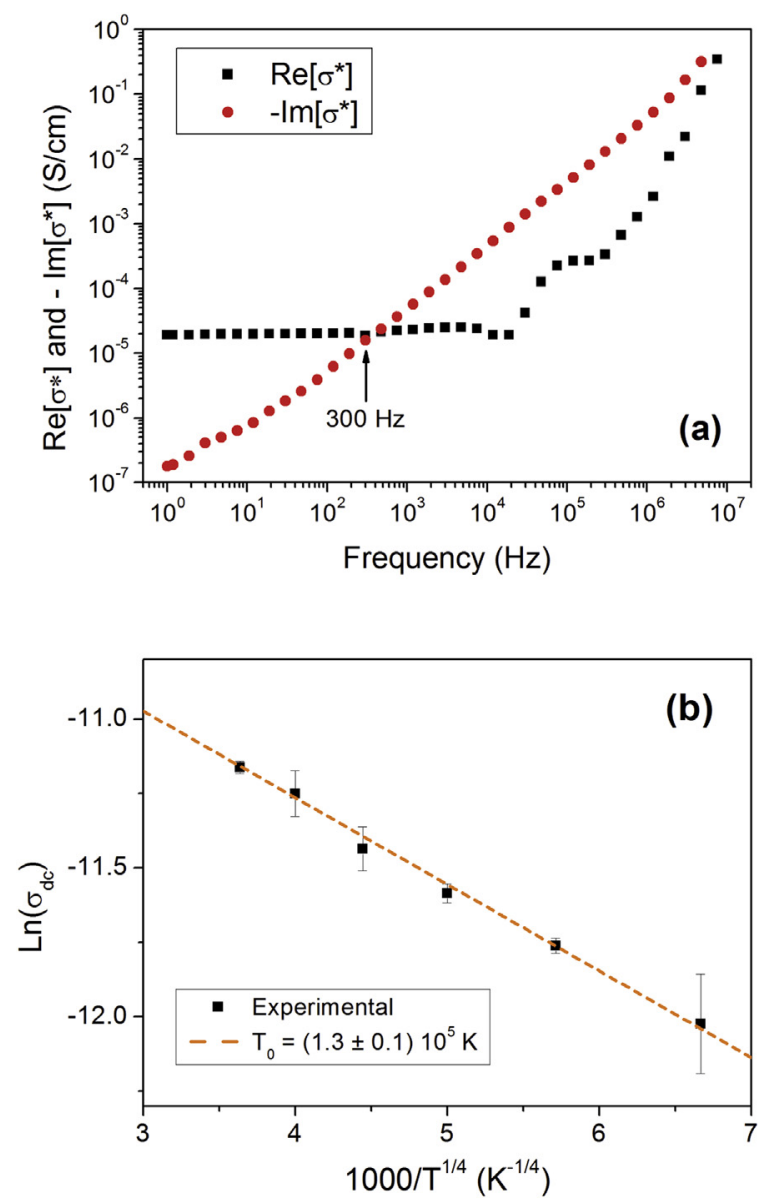

Fig. A.1. Electrical characterization results obtained from the conductive material used as matrix to produce the light-emitting composite. (a) Impedance spectroscopy results; (b) d.c. limit electrical conductivity in the 3D-VRH plot.

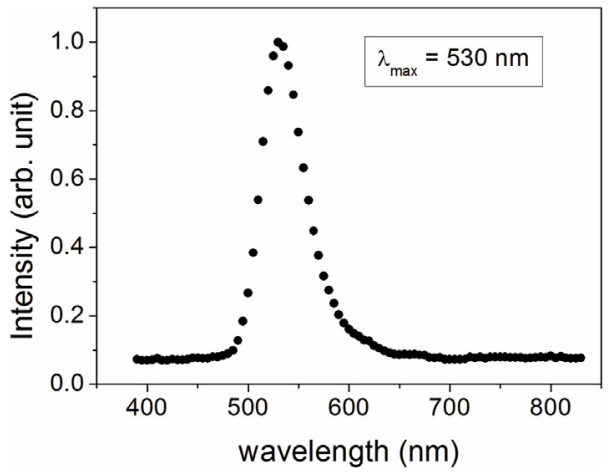

Fig. A.2. Electroluminescence spectra of the light-emitting composite produced with PEDOT:PSS, PVA and $\mathrm{Zn}_{2} \mathrm{SiO}_{4}: \mathrm{Mn}_{\text {. }}$

\section{References}

[1] M.M.G. Destriau, M.J. Perrin, Comptes Rendus des Seances de L'academie des Sciences 208 (1939) 36

[2] M.M.G. Destriau, G. Loudette, Comptes Rendus des Seances de L'academie des Sciences 208 (1939) 5169.

[3] P.D. Rack, P.H. Holloway, Material Science and Engeneering R21 (1998) 171.

[4] P.H. Holloway, T.A. Trottier, B. Abrams, C. Kondoleon, S.L. Jones, J. Vac. Sci. Technol. B 17 (1999) 758.

[5] M.V. Zamoryanskaya, A.N. Trofimov, Optic Spectrosc. 115 (2013) 91.

[6] W.A. Thornton, J. Appl. Phys. 33 (1962) 3045.

[7] K. Brennan, J. Appl. Phys. 64 (1988) 4024.

[8] M.V. Fischetti, et al., Phys. Rev. B 31 (1985) 8124

[9] E. Bringuier, J. Appl. Phys. 70 (1991) 4505.

[10] S. Bhushan, F.S. Chandra, Pramana 24 (1985) 575.

[11] S. Bhushan, F.S. Chandra, J. Phys. D Appl. Phys. 17 (1984) 589.
[12] M.F. Bulanyi, E.V. Kovaleva, B.A. Polezhaev, Inorg. Mater. 37 (2001) 108.

[13] H. Shichijo, K. Hess, Phys. Rev. B 23 (1981) 4197.

[14] E. Bringuier, J. Appl. Phys. 70 (1991) 4505.

[15] D. Arnold, E. Cartier, D.J. DiMaria, Phys. Rev. B 49 (1994) 10278.

[16] A.F. Cattell, J.C. Inkson, J. Kirton, J. Appl. Phys. 61 (1987) 722.

[17] J.M. Blackmore, A.F. Cattell, K.F. Dexter, J. Kirton, P. Lloyd, J. Appl. Phys. 61 (1987) 714.

[18] V. Ceric, Z. Ogorelec, J. Mater. Sci. 7 (1972) 359.

[19] D.H. Smith, J. Lumin. 23 (1981) 209.

[20] R. Myers, J.F. Wager, J. Appl. Phys. 81 (1997) 506.

[21] X. Ouyang, A.H. Kitai, T. Xiao, J. Appl. Phys. 79 (1996) 3229.

[22] J.M. Blackmore, A.F. Cattell, K.F. Dexter, J. Kirton, P. Lloyd, J. Appl. Phys. 61 (1987) 714.

[23] T. Minami, Solid State Electron. 47 (2003) 2237.

[24] T. Minami, H. Yamada, Y. Kubota, T. Miyata, SPIEL 3242 (1997) 229.

[25] A.H. Kitai, Thin Solid Films 445 (2003) 367.

[26] D.L. Chinaglia, et al., Phil. Mag. Lett. 87 (2007) 403. 
[27] K. Janeczek, G. Kozioł, M. Jakubowska, A. Arazna, A. Młozniak, K. Futera, Mater. Sci. Eng. B 178 (2013) 511.

[28] J.G. Tait, et al., Sol. Energy Mater. Sol. Cell. 110 (2013) 98.

[29] P.A. Levermore, et al., J. Mater. Chem. 18 (2008) 4414.

[30] A. Sandström, et al., Nat. Commun. 3 (2012) 1002.

[31] G. Gozzi, D.L. Chinaglia, T.F. Schmidt, O.N. Oliveira Jr., Materials Science \& Engineering. C, Biomimetic Materials, Sensors and Systems C 31 (2011) 969.

[32] G. Gozzi, D.L. Chinaglia, T.F. Schmidt, L.C.M. Walmsley, C.J.L. Constantino, A.E. Job, L.F. Santos, O.N. Oliveira Jr., J. Phys. Appl. Phys. 39 (2006) 3888.

[33] J. Ouyang, et al., Polymer 45 (2004) 8443.

[34] O. Carr, et al., Transl. Mater. Res. 2 (2015) 015002.
[35] C. Chen, et al., Synth. Met. 161 (2011) 2259.

[36] D. Alemu, et al., Energy Environ. Sci. 5 (2012) 9662.

[37] N. Kim, et al., Adv. Mater. 26 (2014) 2268.

[38] U. Lang, et al., Adv. Funct. Mater. 19 (2009) 1215.

[39] T.H. Cho, H.J. Chang, Ceram. Int. 29 (2003) 611.

[40] N.F. Samsudin, et al., J. Spectrosc. (2015) 730753.

[41] T. Minami, H. Yamada, Y. Kubota, T. Miyata, SPIE-3242, Smart Electronics and MEMS 229 (1997) 229.

[42] N.F. Mott, Phil. Mag. 19 (1969) 835.

[43] J.C. Dyre, J. Appl. Phys. 64 (1988) 2456.

[44] Z. Zeng, et al., ACS Appl. Mater. Interfaces 9 (2017) 41078. 